\title{
Institutionalisierungspfade von Naturschutzgebieten Drei Fallbeispiele und ein Modell
}

\section{Monika Röper, Eichstätt}

Die Entstehung und der Verlauf von Konflikten bei der Planung und Einrichtung von Naturschutzgebieten in Entwicklungsländern lassen sich durch die Kombination verschiedener sozialgeographischer Ansätze modellhaft erfassen. Naturschutzgebiete können dabei als eine Form der Institutionalisierung des Zugangs zu und der Kontrolle von Raum begriffen werden. Anhand des Vergleiches von drei Schutzgebieten aus der Pantanal-Region im Westen Brasiliens zeigt sich allerdings, dass der Institutionalisierungsprozess selbst innerhalb eines einheitlichen regionalen Rahmens sehr unterschiedliche Verläufe nehmen kann.

\section{Naturschutzgebiete in Entwicklungsländern: Über die Praxis zur Theorie?}

Die sozialwissenschaftliche Beschäftigung mit Naturschutzgebieten in Entwicklungsländern ist um viele Jahre jünger als das Bestehen eines Großteils der Gebiete selbst: Erst mit der allmählichen Aufnahme von Umweltthemen in die Entwicklungsdiskussion in den 80er Jahren wurden Naturschutzgebiete als sozialwissenschaftliches Forschungsfeld entdeckt. In vielen Entwicklungsländern waren zu diesem Zeitpunkt aber bereits seit mehreren Jahrzehnten Naturschutzgebiete eingerichtet worden. Der eigenständige, von der entwicklungstheoretischen Debatte (aber auch von der entwicklungspolitischen Praxis) zunächst weitgehend losgelöste Entstehungshintergrund der Schutzgebiete führte dazu, dass diese eine Reihe von spezifischen Charakteristika aufweisen, die auch ihre aktuelle Ausgestaltung noch stark beeinflussen. Gleichzeitig zeigten Naturschutzgebiete als Instrumente des Umwelt- und Flächenschutzes aber eine außerordentliche Wandlungsfähigkeit: Sie haben mehrmalige Umdeutungen ihrer Funktionen überstanden und gelten auch derzeit noch als unverzichtbare Bestandteile internationaler und nationaler Naturschutzstrategien (vgl.zum Beispiel das ihnen gewidmete Kapitel in der 1992 unterzeichneten Biodiversitätskonvention: http://www.biodiv.org/ chm/conv/art8.htm).

Doch gerade die Entstehungsgeschichte der Naturschutzgebiete, die sich durch einen starken, nahezu weltweit zu verzeichnenden Einfluss «klassischer» US-amerikanischer Schutzgebietskonzeptionen kennzeichnet (vgl. dazu zum Beispiel GómEz-Pompa \& Kaus 1992, Olwig \& Olwig 1979; zur Charakteri- sierung des «klassischen» Modells vor allem BlaIKIE \& JeanRenaud 1997: 62), hat dazu beigetragen, dass mit ihrer Einrichtung in Entwicklungsländern eine Reihe von Konflikten verbunden waren und sind. Ein zentrales Problem stellt die in der «klassischen» Konzeption verankerte Vorstellung der besonderen Schutzwürdigkeit der vom Menschen «unberührten» Natur dar. Gerade in Entwicklungsländern wurde von vielen Naturschutzideologen allerdings nicht erkannt, dass es sich dabei häufig um kulturlandschaftliche Artefakte handelte, denen komplexe Nutzungssysteme zugrundelagen. Aus dem Totalschutzmodell wurde die Notwendigkeit abgeleitet, ab dem Zeitpunkt der Unterschutzstellung die bis dahin bestehenden Zugangsund Nutzungsformen des Gebietes zu verhindern respektive zu beseitigen, wobei dem Staat bei der oft mit Gewalt erfolgten Durchsetzung dieser Vorgaben eine zentrale Rolle zukam. Eingeführt und gefördert werden sollten dagegen Nutzungsformen, von denen angenommen wird, dass sie die Integrität der Natur nicht gefährden (wie zum Beispiel Besuchsaktivitäten und Forschung). Vor diesem Hintergrund ergab sich eine große Anzahl zum Teil gravierender Konflikte, vor allem mit den Betroffenen der Schutzgebietseinrichtung auf lokaler Ebene. Deren Versuche, die Schutzgebietspläne zu bekämpfen oder zu unterlaufen, haben schließlich zunehmende Kritik an der Wirksamkeit und Durchsetzbarkeit, aber auch an der moralischen Vertretbarkeit der «klassischen» Konzeptionen laut werden lassen (vgl. zum Beispiel GHimire \& PIMBERT 1997, COLCHESTER 1997).

Spätestens seit den 80er Jahren ist jedoch ein Wandel in der Wahrnehmung der Rolle von Bewohnern und Nutzern für die Schutzgebiete zu verzeichnen. Mittlerweile hat sich auf internationaler Ebene ein Naturschutzverständnis durchgesetzt, bei dem von der gegenseitigen Ergänzung von streng geschützten Gebieten und solchen, die ausgewählte Nutzungsformen und die Anwesenheit von Bewohnern zulassen, ausgegangen wird (vgl. IUCN 1994). Dennoch ist das Totalschutzmodell US-amerikanischer Prägung gerade in vielen Entwicklungsländern sowohl als idealtypisches Leitbild als auch in der Praxis noch vielfältig wirksam.

Im Text gelten personenbezogene Bezeichnungen als geschlechtsneutral. 
Mit dem erwähnten Wandel wurden Forderungen nach einer intensiveren sozialwissenschaftlichen $\mathrm{Be}$ schäftigung mit der Schutzgebietsthematik laut. Entsprechende Ansätze sind allerdings in der Regel durch eine ausgesprochene Anwendungsorientierung gekennzeichnet, in denen Schutzgebiete weniger als Gegenstand theoretischer Überlegungen dienen, sondern meist anhand von Fallbeispielen in Bezug auf ihre Ausgestaltung und Auswirkungen untersucht werden. Diese Diagnose gilt auch für die geographische Auseinandersetzung mit Schutzgebietsfragen in Entwicklungsländern: Obwohl eine Reihe von Fallstudien zum Beispiel die Bezüge zwischen Schutzgebietseinrichtungen, Flächennutzungskonflikten, Ökotourismus, Regionalentwicklungsvorhaben oder Entwicklungszusammenarbeit untersuchen (vgl. ELLENBERG 1998), sind eher analytisch orientierte, an der Hinterfragung der zugrunde gelegten Schutzkonzepte ausgerichtete Arbeiten erst in jüngster Zeit anzutreffen (vgl. zum Beispiel MÜLLER-BÖKER, BACKhaus \& Kollmair 1998). Dieser neue, sozialgeographisch geprägte Blick auf Naturschutzgebiete leitet sich zum einen aus der Erkenntnis ab, dass die in der Praxis registrierten Konflikte in den zugrunde gelegten Konzeptionen des Naturschutzes selbst zu suchen sind. Ein weiterer, damit verbundener und für die geographische Analyse interessanter Auslöser konfliktträchtiger Entwicklungen, dem in diesem Beitrag nachgegangen werden soll, ist darin zu sehen, dass mit einem Naturschutzgebiet die Institutionalisierung eines bis dahin zumindest in dieser Form nicht existenten Raumausschnittes mit sehr spezifischen Funktionszuschreibungen verbunden ist.

\section{Die Institutionalisierung von Naturschutzgebieten in sozialgeographischer Betrachtung}

In den folgenden Ausführungen soll also davon ausgegangen werden, dass Naturschutzgebiete selbst eine Form der Institutionalisierung des Zugangs zu und der Kontrolle von Raum darstellen. Um die Entstehung der dabei auftretenden Konflikte genauer zu erfassen und zu charakterisieren sollen einige Bestandteile von BENNO WERLENs «Sozialgeographie der alltäglichen Regionalisierungen» auf die Planung und Einrichtung von Naturschutzgebieten übertragen werden. Der Einrichtungsprozess soll dementsprechend als Herstellungsprozess eines sozialen und politischen Raumes verstanden werden. In WeRLENs Typologie der «alltäglichen Regionalisierungen» lässt er sich den sogenannten normativ-politischen Regionalisierungen zuordnen (vgl. WerLEN 1997: 271ff.). Diese setzen sich einerseits mit akteurs- beziehungsweise gruppenspezifischen Aneignungsformen des Raumes, andererseits mit deren gesellschaftlicher Koordination auseinander. Die Tatsache, dass die ökonomisch, sozio-kulturell, symbolisch und durch symbolische Zuschreibungen und die jeweils verfügbaren Informationen geprägten Aneignungsformen des Raumes intersubjektiv nicht einheitlich sind und zudem dem zeitlichen Wandel unterliegen, führt dazu, dass räumliche Zugangsrechte und Entscheidungsbefugnisse gesellschaftlich festgelegt und durchgesetzt werden müssen. Diese Festlegungen müssen schliesslich im Verlauf gesellschaftlicher und politischer Wandlungsprozesse immer wieder überprüft und gegebenenfalls revidiert werden. Auf die Einrichtung von Naturschutzgebieten lassen sich die zwei Komponenten normativ-politischer Regionalisierungen in folgender Weise übertragen:

- Die unterschiedlichen Wahrnehmungen und Wertungen von Natur können als spezifische Form der Raumaneignung verstanden werden. Aus den Naturwahrnehmungen verschiedener Akteursgruppen und Individuen leiten sich unterschiedliche Vorstellungen zur Notwendigkeit und zur Ausgestaltung des Schutzes von Natur ab.

- Die konkreten Schritte der Planung und Einrichtung der Gebiete stellen eine Form der politischen Kontrolle des Raumes dar: In dieser Etappe entscheidet sich, welche Gruppen letztlich ihre Vorstellung des Naturschutzes sowohl konzeptionell als auch diskursiv und praktisch gegen konkurrierende Konzepte durchsetzen können.

Die von WERLEN vorgenommene Unterscheidung zwischen alltäglichen, wissenschaftlichen und administrativen Regionalisierungen (vgl. WerLen 1997: 46f.) erweist sich für die Analyse von Naturschutzgebieten ebenfalls als relevant. Der Einrichtungsprozess eines Naturschutzgebietes besteht zu einem wichtigen Teil aus administrativen Regionalisierungen, das heisst solchen, die von juristischen und politischen Organisationen und Institutionen bestimmt werden. Allerdings werden diese in ihrer Umsetzung sowohl von den alltäglichen Regionalisierungen der in den Organisationen agierenden Individuen als auch von anderen betroffenen und beteiligten Akteuren beeinflusst. Auch die Trennung in alltägliche und wissenschaftliche Regionalisierungen kann im Naturschutz letztlich nur in analytischer Hinsicht aufrechterhalten werden. Wissenschaftliche Regions- und Naturkonzepte üben bei der Einrichtung von Naturschutzgebieten zwar zentrale handlungsleitende Funktionen aus, sie werden aber auch gezielt zur Untermauerung von Handlungsund Diskursstrategien zur Durchsetzung des Naturschutzes eingesetzt, wobei sie sich auf der Ebene der alltäglichen Handlungen häufig mit anderen Regionalisierungstypen vermischen.

Betont werden muss dabei nochmals, dass sich die Auseinandersetzungen zwischen den unterschiedlichen Naturwahrnehmungen, Interessen und Akteuren nicht in einem Machtvakuum vollziehen, sondern dass ein großer Teil der verzeichneten Konflikte auf die zum Teil bis heute dominierende Stellung der erwähnten 
«klassischen» Naturschutz-Konzepte und seine Verfechter zurückzuführen ist.

Die einzelnen Etappen der Institutionalisierung von Naturschutzgebieten weisen weiterhin große Parallelen zu der von ANSSI PAASI (1986) vorgenommenen Aufgliederung der Institutionalisierung von Regionen in unterschiedliche Phasen auf:

- Zum Ausschluss nicht erwünschter Nutzungsformen wird eine klare juristische Grundlage benötigt, in anderen Worten, eine Schutzgebietsgesetzgebung. Für die Durchsetzung der Schutzvorgaben ist es notwendig, ihren Geltungsbereich räumlich eindeutig abzugrenzen, das heisst eine territoriale Form für das Schutzgebiet zu definieren.

- Aufgrund der ihnen zugesprochenen Bedeutung für das Allgemeinwohl wird den Schutzgebieten der Status von öffentlichen Gütern zugewiesen, der die Basis für die Rolle des Staates als Eigentümer und Verwalter der Gebiete liefert. Dies bildet wiederum die Grundlage zur Etablierung institutioneller Formen in Gestalt von Organisationen und Regeln zur Schutzgebietsverwaltung.

- Schliesslich kommt es bei der Einrichtung von
Naturschutzgebieten auch zur Schaffung von raumbezogenen symbolischen Formen, die materielle Identifikationsobjekte (Logos, Grenzmarkierungen, Verwaltungsgebäude etc.) umfassen können, bei denen aber auch PAASIs eher semiotisch angelegte Interpretation (die sich zum Beispiel in der Namensgebung für das Gebiet widerspiegelt) eine Rolle spielt (vgl. PaAsI 1986: 125).

Die hier vorgestellten Grundkomponenten lassen jedoch keineswegs auf einen einheitlichen Ablauf der Institutionalisierung von Naturschutzgebieten schließen. Eine der Schwierigkeiten des Umgangs mit den durch die Schutzgebietseinrichtung hervorgerufenen Konflikten liegt gerade darin begründet, dass bei ihrer Planung und Einrichtung in der Regel ein Zusammenwirken zahlreicher Akteure, Interessen, Aneignungs- und Nutzungsmustern, Handlungsfeldern und Maßstabsebenen zu verzeichnen ist. Dass sich selbst innerhalb eines einheitlichen regionalen Rahmens sehr unterschiedliche Verläufe ergeben können, soll im Folgenden anhand des Vergleiches der Institutionalisierung von drei Naturschutzgebieten der im Westen Brasiliens gelegenen Pantanal-Region gezeigt werden.

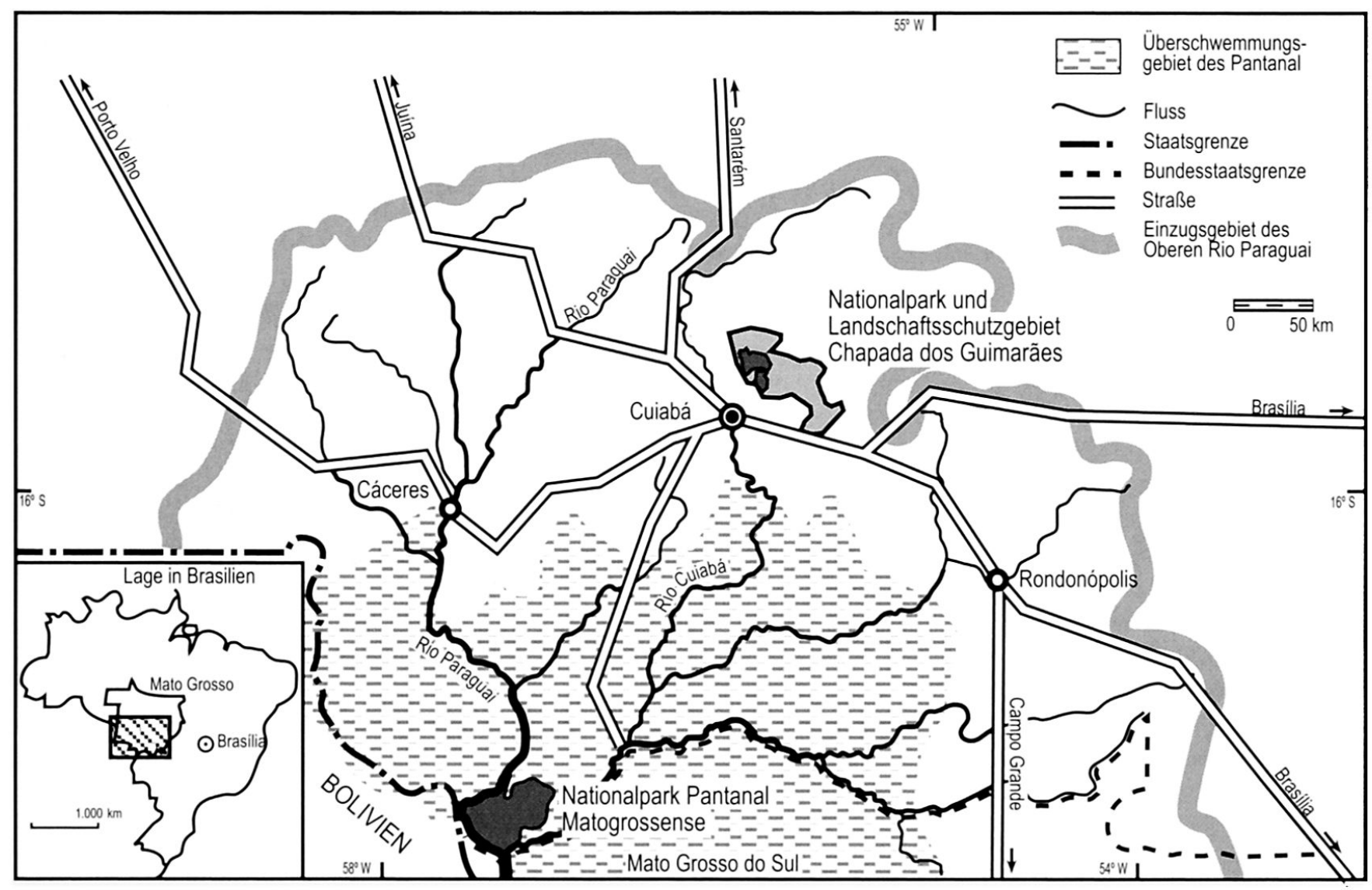

Abb. 1: Lage der Fallbeispiele in der Pantanal-Region Localization of the case studies in the Pantanal-Region Localisation des exemples dans le bassin du Pantanal Kartengrundlage: CoY 1991: 180; Entwurf und Bearbeitung: M. RÖPER 


\section{Naturschutzgebiete in der Pantanal-Region: kurze Charakterisierung der Fallbeispiele}

Alle hier betrachteten Fallbeispiele liegen in dem im brasilianischen Bundesstaat Mato Grosso gelegenen Teil des Einzugsgebietes des Pantanal. Das Pantanal ist ein quartäres Aufschüttungsgebiet, das von periodischen Überschwemmungen geprägt wird und das aufgrund seiner Größe (ca. 140'000 km²) und seiner üppigen Tier- und Pflanzenwelt als Feuchtgebiet von internationaler Bedeutung gilt (vgl. Abb. 1). Die Überschwemmungsdynamik wird dabei durch komplexe Abhängigkeiten der Pantanal-Ebene von den umliegenden, zum zentralbrasilianischen Bergland gehörenden Hochflächen gesteuert, auf denen die Pantanal-Quellflüsse entspringen. Gerade auf den Hochflächen wurden aber durch die in den 60er Jahren einsetzende Inkorporation der Binnenräume
Zentralbrasiliens eine Reihe von Entwicklungen ausgelöst - modernisierte Landwirtschaft, Infrastrukturausbau, rasante Verstädterung usw. -, die sowohl das ursprünglich durch Savannenformationen bedeckte Einzugsgebiet als auch das sensible Ökosystem des Pantanal zunehmend gefährden (vgl. Coy 1991). Die Einrichtung der in der Region existierenden Schutzgebiete steht allerdings eher mit einer Reihe von Zufallsfaktoren als mit den naturräumlichen Gegebenheiten in Verbindung, denn erst in jüngster Zeit sind Bemühungen zur Erarbeitung systematischer Schutzstrategien zu verzeichnen.

Der folgende Vergleich von drei Naturschutzgebieten basiert auf intensiven Feldarbeiten in der Region. Dabei ist zu beachten, dass die zuvor vorgestellten theoretischen Ansätze aufgrund ihres hohen Abstraktionsgrades und einiger weiterer Einschränkungen eine unmittelbare Anwendung im Rahmen empirischer Analysen

\begin{tabular}{|c|c|c|c|}
\hline & $\begin{array}{l}\text { Ökologische Station } \\
\text { Serra das Araras }\end{array}$ & $\begin{array}{c}\text { Nationalpark } \\
\text { Chapada dos Guimarães }\end{array}$ & $\begin{array}{c}\text { Nationalpark } \\
\text { Pantanal Matogrossense }\end{array}$ \\
\hline Einrichtungsjahr, Größe & $1982,28.700$ ha & $1989,33.000$ ha & $1981,135.000 \mathrm{ha}$ \\
\hline Hauptfunktionen & Integraler Schutz, Forschung & Landschaftsschutz, Tourismus & Landschaftsschutz, Tourismus \\
\hline Motiv der Einrichtung & $\begin{array}{l}\text { Schutz eines «unberührten» Tales. } \\
\text { Die Fläche wurde nach einer } \\
\text { einmaligen Sichtung bei einem } \\
\text { Überflug ausgewählt. }\end{array}$ & $\begin{array}{l}\text { Forderungen lokaler NGOs nach } \\
\text { Schutz des zum Pantanal hin ab- } \\
\text { fallenden Stufenhangs und seiner } \\
\text { Umgebung. Durch Berücksichti- } \\
\text { gung von Spekulationsinteressen } \\
\text { wurde aber nur ein Teil der geplan- } \\
\text { ten Fläche als Park eingerichtet. }\end{array}$ & $\begin{array}{l}\text { Erstes Schutzgebiet im Über- } \\
\text { schwemmungsbereich des Panta- } \\
\text { nal. Die Flächenwahl orientierte } \\
\text { sich aber an der Verfügbarkeit von } \\
\text { preiswertem Land. }\end{array}$ \\
\hline Einrichtungsproze $\beta$ & $\begin{array}{l}\text { Ausweisung der kleinbäuerllichen } \\
\text { Bewohner, nur zum Teil } \\
\text { Entschädigung, dagegen Aner- } \\
\text { kennung von Invasionen durch } \\
\text { Großgrundbesitzer }\end{array}$ & $\begin{array}{l}\text { Schleppende Enteignung nach } \\
\text { erratischen Kriterien, Kritik } \\
\text { an der Demarkation durch NGOs, } \\
\text { Forderungen nach Parkerweite- } \\
\text { rung, nachträgliche Invasionen } \\
\text { (v.a. Wochenendgrundstücke). }\end{array}$ & $\begin{array}{l}\text { Kritik an der Lage und Abgren- } \\
\text { zung des Parks, Forderungen } \\
\text { nach Parkerweiterung durch } \\
\text { Wissenschaftler und NGOs. }\end{array}$ \\
\hline $\begin{array}{l}\text { Ausstattung und } \\
\text { Management }\end{array}$ & $\begin{array}{l}\text { Personalmangel, Infrastruktur } \\
\text { verfällt, kein Managementplan. } \\
\text { Aktuell keine akuten Konflikte, } \\
\text { Station wird jedoch kaum genutzt. }\end{array}$ & $\begin{array}{l}\text { Personalmangel, Infrastruktur mit } \\
\text { internationalen Mitteln finanziert, } \\
\text { «Not-Aktionsplan» als Manage- } \\
\text { mentinstrument. Teilweise eigene } \\
\text { Managementaktionen durch } \\
\text { NGOs. }\end{array}$ & $\begin{array}{l}\text { Personalmangel, Infrastruktur mit } \\
\text { internationalen Mitteln finanziert, } \\
\text { «Not-Aktionplan» als Manage- } \\
\text { mentinstrument, Einrichtung } \\
\text { privater Schutzgebiete zur } \\
\text { Erweiterung des Parks durch } \\
\text { NGOs }\end{array}$ \\
\hline $\begin{array}{l}\text { Beteiligte und } \\
\text { betroffene Akteure }\end{array}$ & $\begin{array}{l}\text { Kleinbauern, Großgrund- } \\
\text { besitzer, Anrainer }\end{array}$ & $\begin{array}{l}\text { Bewohner (Groß- und Klein- } \\
\text { betriebe, Wochenendgrundstücke } \\
\text { etc.), Anrainer, NGOs, bundes- } \\
\text { staatliche und lokale Behörden, } \\
\text { Besucher, Tourismus- und } \\
\text { Immobilienbranche }\end{array}$ & $\begin{array}{l}\text { NGOs, Anrainer, Tourismus- } \\
\text { fischer: durch die geringe Präsenz } \\
\text { der Parkverwaltung vor Ort in der } \\
\text { Praxis kaum Interaktionen }\end{array}$ \\
\hline $\begin{array}{l}\text { Parallele } \\
\text { regionale Prozesse }\end{array}$ & $\begin{array}{l}\text { Wandel des ländlichen Raumes: } \\
\text { neue Akteure, Landkonflikte, } \\
\text { Verdrängungsprozesse }\end{array}$ & $\begin{array}{l}\text { Verstädterung, Wandel des ländli- } \\
\text { chen Raumes, Einflußnahme } \\
\text { gesellschaftlich wichtiger Gruppen }\end{array}$ & $\begin{array}{l}\text { Niedergang traditioneller } \\
\text { Nutzungssysteme im Pantanal, } \\
\text { Tourismusbranche als neue } \\
\text { Akteursgruppe }\end{array}$ \\
\hline $\begin{array}{l}\text { Parallele } \\
\text { nationale Prozesse }\end{array}$ & \multicolumn{3}{|c|}{$\begin{array}{l}\text { Krise und Restrukturierung der Umweltbehörden im Rahmen von Dezentralisierungsprozessen. } \\
\text { Zunahme der Finanzierung von Regional- und Umweltpolitik durch internationale Programme. } \\
\text { Engagement von NGOs, Einbindung der Planungsbetroffenen, partizipative Prozesse. }\end{array}$} \\
\hline
\end{tabular}


in Entwicklungsländern nur bedingt zulassen. Daher wurden sie vor allem durch die im spezifischen Kontext der Entwicklungsdebatte entstandenen Ansätze der politischen Ökologie ergänzt (vor allem BRYANT \& Bailey 1997, Peet \& Watts 1996, Blaikie 1995).

An dieser Stelle sollen die ausgewählten Fallbeispiele aber nur sehr kurz mit Hilfe von Abb. 2 charakterisiert werden (für eine etwas ausführlichere Darstellung vgl. Röper 1998, 2000). Hervorzuheben ist, dass mit ihnen jeweils unterschiedlich geprägte Konstellationen erfasst werden: So sind sowohl sehr entlegene (Nationalpark Pantanal Matogrossense) als auch stadtnahe (Nationalpark Chapada dos Guimarães) Gebiete vertreten, ein Umstand, der sich vor allem auf die Zahl der beteiligten Akteure und Konflikte auswirkt. Das Spektrum konkreter Ausgestaltungen spiegelt sich auch in den vertretenen Schutzkategorien wider (für die der Anschaulichkeit halber deutschsprachige Bezeichnungen verwendet werden), die jeweils unterschiedliche planerische Ansätze und Möglichkeiten der praktischen Umsetzung repräsentieren.

Als zentrales Ergebnis der empirischen Erhebungen bleibt noch herauszustellen, dass sowohl ein unmittelbar mit der Schutzgebietsthematik zusammenhängender Komplex von Themen und Handlungsfeldern, als auch eine Reihe von parallelen und indirekten Einflussfaktoren von entscheidender Bedeutung für den Erfolg oder das Scheitern der Vorhaben sind. Alle diese Prozesse und Faktoren werden letztlich erst über die Handlungen der beteiligten und betroffenen Akteursgruppen wirksam.

\section{Konsolidierung, Konflikt, Auflösung, Interaktion: vier Pfade der Institutionalisierung}

Die Gründe für die in der Pantanal-Region verzeichneten, überwiegend krisenhaften Entwicklungen in den Naturschutzgebieten sollen im Folgenden mit Hilfe der zuvor skizzierten theoretischen Ansätze näher betrachtet werden. In Abb. 3 wird hierzu der unterschiedliche Verlauf der Institutionalisierungspfade in den Fallbeispielen rekonstruiert (Szenarien 1 bis 3). Ihnen soll allerdings auch ein möglicher alternativer Verlauf gegenübergestellt werden, in dem neuere konzeptionelle Entwicklungen aus der internationalen und brasilianischen Debatte um Naturschutzgebiete sowie Erfahrungen aus Pilotprojekten aufgegriffen und modellhaft umgesetzt werden (Szenario 4).

Ausgangspunkt der Überlegungen ist die Zuordnung der Planung und Einrichtung von Naturschutzgebieten zu den normativ-politischen Regionalisierungen. Wie zuvor dargestellt, lassen sich diese in zwei Etappen zerlegen: in die Aneignung des Raumes und in seine politische Kontrolle.

Die Phase der Raumaneignung verläuft bei allen bestehenden Naturschutzgebieten weitgehend einheitlich.
Sie ist in erster Linie dadurch charakterisiert, dass die verantwortlichen Planer nach (natur-) wissenschaftlich abgesicherten Begründungen für die Schutzgebietsvorhaben suchen. Dies gilt sogar, wenn die anschließende Umsetzung der Pläne durch erratische und intuitive Vorgehensweisen geprägt wird, wie es in der Untersuchungsregion überwiegend der Fall war. In der Regel leiten die Begründungen der Schutzwürdigkeit eines Gebietes auch die Definition der territorialen Form, die das zukünftige Naturschutzgebiet annehmen soll, sowie seine institutionelle Reglementierung, die als wichtige Komponente zum Beispiel die Wahl einer geeigneten Schutzkategorie beinhaltet, aus der sich im weiteren Verlauf bereits eine Reihe von Vorgaben zur Ausgestaltung und Verwaltung des Gebietes ergeben. Bei allen hier betrachteten Fallbeispielen war mit den gewählten Schutzkategorien die Enteignung und Ausweisung der Bewohner aus den Gebieten verbunden. Die Umsetzung dieser Massnahmen verlief allerdings in den drei Schutzgebieten sehr unterschiedlich (vgl. Abb. 2).

In der Regel setzt erst ab dem Zeitpunkt der juristischen Ausweisung des Naturschutzgebietes ein intensiverer Prozess der Auseinandersetzung mit den in dem gewählten Raumausschnitt bereits bestehenden Regionalisierungsformen ein, die in Form von Szenarien näher betrachtet werden sollen. Vereinfachend sind dabei vier grundlegende Regionalisierungsformen zu unterscheiden (vgl. Szenario 1 in Abb. 3):

a) Administrative Regionalisierungen (zum Beispiel Verwaltungsgrenzen), die in der Regel zum Zeitpunkt der Schutzgebietseinrichtung bereits bestehen und daher überlagert werden.

b) Konsolidierte alltägliche Regionalisierungen: Dabei handelt es sich um bestehende Regionalisierungen, die bis zum Zeitpunkt der Einrichtung nicht durch andere Regionalisierungen angefochten oder in Frage gestellt wurden. Die Nutzungssysteme, die auf der Grundlage dieses Regionalisierungstyps entstehen, erweisen sich als funktionsfähig und die $\mathrm{Zu}$ gangs- und Entscheidungsrechte der sie ausübenden Akteursgruppen sind gesichert.

c) In Auflösung befindliche Regionalisierungen: Unter diesem Begriff sollen Regionalisierungstypen zusammengefasst werden, die nicht mehr in einer zuvor bestehenden Form aufrecht erhalten werden können. Dabei sind ganz unterschiedliche Auslöser für diesen Zustand vorstellbar, wie zum Beispiel Konflikte mit den Verfechtern anderer Regionalisierungsformen, veränderte Rahmenbedingungen, Krisen der Nutzungssysteme etc. Der Auflösungsprozess geht mit einem Verlust von Zugangsund Entscheidungsbefugnissen einher.

d) In Konsolidierung befindliche Regionalisierungen: Damit sollen neu entwickelte oder eingeführte Regionalisierungsformen bezeichnet werden. Die sie verfechtenden Akteure sind im Begriff, sich 


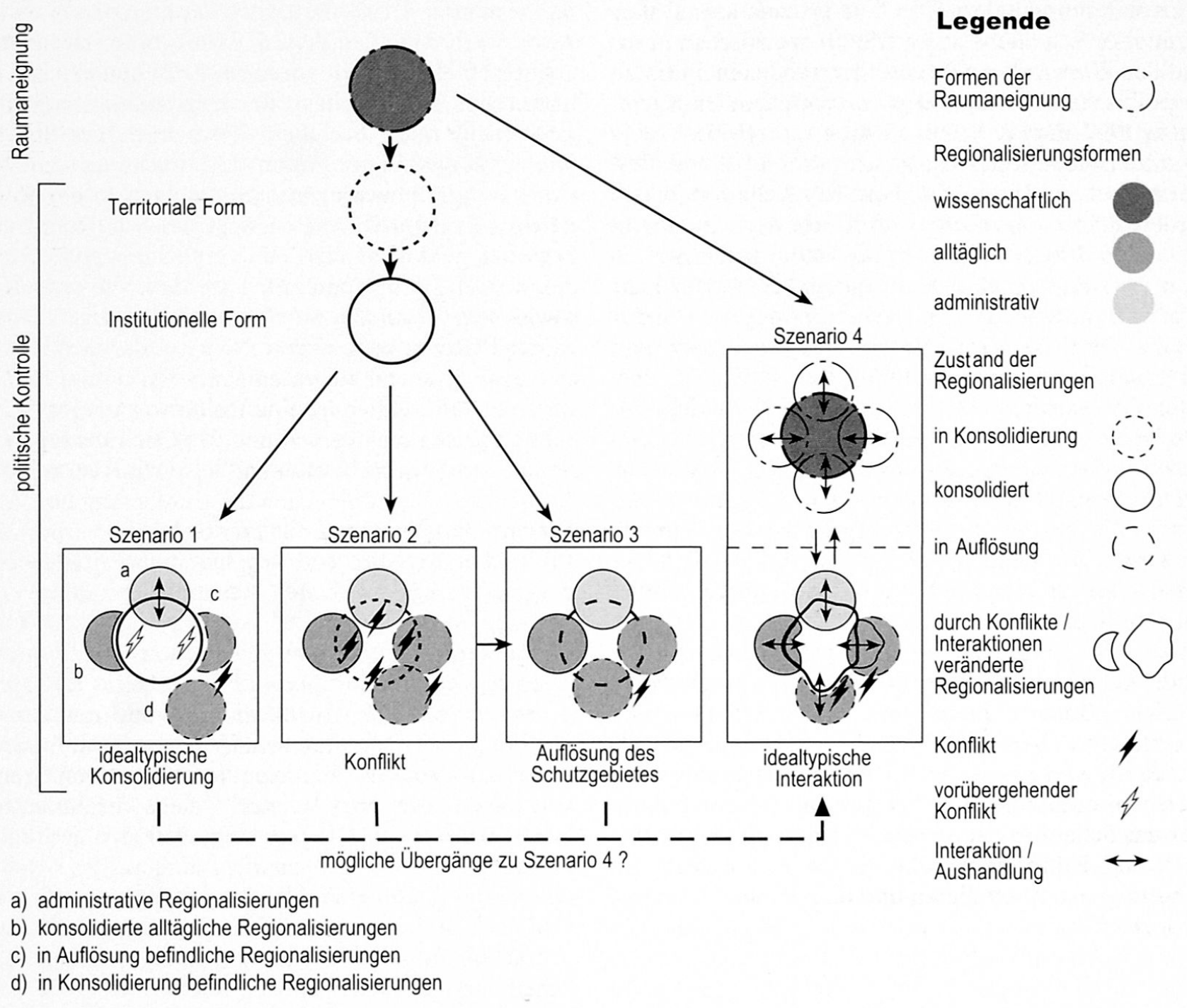

Abb. 3: Institutionalisierungspfade der Planung und Einrichtung von

M. RÖPER 2000

Naturschutzgebieten

Institutionalization paths in the planning and the implementation of protected areas

La création de réserves naturelles et les voies de son institutionalisation

erfolgreich gegen konkurrierende Regionalisierungsformen durchzusetzen.

Die in der Realität bestehenden vielfältigen Überlagerungen und Interaktionen zwischen diesen Grundtypen sollen hier aus Gründen der Darstellbarkeit weitgehend unberücksichtigt bleiben. Die folgenden Szenarien beziehen sich daher vorrangig auf Interaktionen zwischen den bestehenden Regionalisierungsformen und der Schutzgebietseinrichtung (vgl. Abb. 3).

\section{Szenario 1: Idealtypische Konsolidierung}

Dieses Szenario entspricht der Entwicklung, die mit dem «klassischen» Schutzgebietsmodell kompatibel wäre, die aber auch der Mehrzahl der Planer auf regionaler Ebene als wünschenswert vorschwebt. Das Naturschutzgebiet ist hier Ausdruck einer konsolidierten Regionalisierungsform. Der naturwissenschaftlich begründete Schutz des Gebietes beinhaltet eine Zurückdrängung oder Auslagerung der bis zum Zeitpunkt der Schutzgebietseinrichtung bestehenden alltäglichen Regionalisierungsformen und der auf ihnen basierenden Nutzungssysteme. Temporäre Konflikte mit den betroffenen Akteursgruppen sind bei der Durchsetzung der Regionalisierungsform zwar möglich, doch die Schutzgebietseinrichtung ist juristisch eindeutig abgesichert, so dass ihre Durchsetzung selbst unter Zuhilfenahme repressiver Mittel formal legitim ist. Nach der Einrichtungsphase werden keine weite- 
ren Konflikte mehr erwartet. Die Interaktion mit den Verantwortlichen für die überlagerten administrativen Regionalisierungsformen gestaltet sich problemlos. Im Umfeld bestehende Konflikte zwischen anderen Regionalisierungsformen haben in diesem Szenario keine Auswirkungen auf das Naturschutzgebiet. Zumindest in der Pantanal-Region ist diese Konstellation jedoch als Idealtypus anzusehen. Am ehesten entspricht dieser Idealvorstellung noch die ökologische Station Serra das Araras in ihrem heutigen Zustand. Eine «Vorbildfunktion» des Einrichtungsprozesses dieser Station wird allerdings durch die gewaltsame und entschädigungslose Vertreibung der zuvor in dem Gebiet ansässigen kleinbäuerlichen Gemeinschaft ausgeschlossen (die allerdings nur zum Teil den Naturschutzbehörden anzulasten ist, vgl. dazu auch RÖPER 1998: 249-252).

\section{Szenario 2: Konflikt}

Dieses Szenario entspricht vor allem dem Fallbeispiel des Nationalparks Chapada dos Guimarães (vgl. auch RÖPER 1998: 252-256). Hier ist zwar ein gewisser Konsolidierungsprozess des Schutzgebietes zu beobachten, doch seine Institutionalisierung wird durch alle anderen präsenten Regionalisierungsformen angefochten. Die zwischen den sonstigen Regionalisierungsformen bestehenden Konflikte wirken sich zusätzlich problemverstärkend auf das Naturschutzgebiet aus. Den Behörden gelingt es letztlich nicht, die Regionalisierung als Naturschutzgebiet durchzusetzen, obwohl das Gebiet juristisch und institutionell unanfechtbar ist. Vor der Einrichtung bestehende Nutzungsformen werden weiter fortgesetzt und stellen zumindest teilweise die Naturschutzziele in Frage. Aufgrund der vielfältigen Interessenkonflikte und der unterschiedlichen Machtzugänge der beteiligten Akteure kann sich schließlich selbst die Interaktion mit den für die überlagerten administrativen Regionalisierungsformen Verantwortlichen konfliktträchtig gestalten, etwa wenn auch lokale Behörden die Existenz und die Ziele der Naturschutzgebiete in Frage stellen.

\section{Szenario 3: Auflösung des Naturschutzgebietes}

Zunächst sei betont, dass mit Auflösung hier keineswegs die juristische Aufhebung des Schutzstatus gemeint ist, sondern seine Auflösung als konkret wahrnehmbare territoriale Form, letztlich also seine Verwandlung in einen der in Lateinamerika so häufig anzutreffenden paper parks, deren Existenz sich oft weitgehend auf das Einrichtungsdekret beschränkt. Die anderen Regionalisierungsformen bestehen dabei weiter, als ob es das Naturschutzgebiet nicht gäbe. Obwohl das Gebiet juristisch und administrativ fortbesteht, findet hier keine Auseinandersetzung mit den unterschiedlichen Regionalisierungsformen vor Ort statt. Das Zustandekommen dieses Szenarios ist auf mehreren Wegen vorstellbar: Zum einen kann es sich dabei um ein fortgeschrittenes Stadium des Konfliktszenarios handeln, in dem die Anfechtung des Naturschutzgebietes so weit vorangeschrittenen ist, dass die konkreten Ausprägungen der Regionalisierung praktisch nicht mehr bestehen. Doch auch ein Beispiel wie der Nationalpark Pantanal Matogrossense, in dem die Institutionalisierungsanstrengungen insgesamt so geringe Ergebnisse zeigten, dass das Schutzgebiet im regionalen Kontext eine fast vernachlässigbare Rolle spielt, kann hier eingeordnet werden. Ob sich diese Auflösungstendenzen durch einen seit einigen Jahren in der Planung befindlichen, völlig neuartigen Ansatz der gemeinsamen Verwaltung des Parks und mehrerer in seiner Umgebung eingerichteter privater Naturschutzgebiete aufhalten lassen, lässt sich derzeit noch nicht abschließend beurteilen (vgl. dazu RÖPER 2000).

\section{Szenario 4: Idealtypische Interaktion}

Im letzten Szenario soll den hier untersuchten Fallbeispielen ein möglicher alternativer Ablauf der Planung und Einrichtung von Naturschutzgebieten gegenübergestellt werden. Seine einzelnen Elemente ergeben sich aus der Auswertung neuerer und innovativer Ansätze des Schutzgebietsmanagements, die auch in Brasilien in unterschiedlichen Pilotprojekten bereits zur Anwendung kommen und weiterentwikkelt werden (vgl. zum Beispiel Ramos \& CAPOBIanco 1996, Drumond 1999). Einige Erfahrungen aus diesen Projekten, die vor allem auf partizipativen, dynamischen und graduellen Planungsprozessen aufbauen, sind auch in die im Jahr 2000 verabschiedete Reform der brasilianischen Schutzgebietsgesetzgebung eingeflossen und werden damit in Zukunft als neue Rahmenbedingungen die weitere Entwicklung der Gebiete beeinflussen (vgl. MMA 2000).

Das Szenario unterscheidet sich bereits dadurch grundlegend von den vorangegangenen, dass die Auseinandersetzung mit den bestehenden Regionalisierungsformen nicht erst nach der Festlegung des Institutionalisierungspfades einsetzt, sondern schon zu seinem Beginn. Die wissenschaftlichen Regionalisierungen, die den Schutz des Gebietes rechtfertigen, fließen dabei in einen Aushandlungsprozess ein, in dem auch andere Regionalisierungsformen zur Kenntnis genommen werden, die das Ergebnis des Prozesses beeinflussen können. Es ist daher wahrscheinlich, dass die aus den Verhandlungen resultierende territoriale Form des Schutzgebietes von den wissenschaftlichen Vorgaben abweicht. Gerechtfertigt wird dies dadurch, dass mit einer höheren Akzep$\operatorname{tanz}$ der ausgehandelten Umrisse durch alle Beteiligten gerechnet werden kann.

Wichtig ist, dass in den Aushandlungsprozessen tatsächlich möglichst alle für die Regionalisierungsformen verantwortlichen Akteursgruppen berücksichtigt werden, und keineswegs nur die Gruppen, deren Aktivitäten von den Schutzgebietsbehörden als mit den Schutzzielen «vereinbar» angesehen werden. Denn es hat 
sich gezeigt, dass der Ausschluss von Akteuren auf Dauer nur dazu beiträgt, dass diese andere Strategien entwickeln, ihre jeweiligen Interessen durchzusetzen. Es muss betont werden, dass dieser Ansatz nicht die Vermeidung von Konflikten zum Ziel hat, sondern ihre aktive Einbeziehung in die Aushandlung: Konflikte sollen grundsätzlich als Bestandteil der Interaktionen verstanden werden.

Auch in diesem Szenario besteht die Möglichkeit, dass bestimmte Regionalisierungsformen und Nutzungssysteme letztlich aus dem Schutzgebiet ausgeschlossen werden. Ebenso kann damit gerechnet werden, dass einige der betroffenen Akteursgruppen sich diesem Beschluss widersetzen beziehungsweise versuchen, ihn zu unterlaufen. Doch selbst bei einem eventuell notwendigen Abbruch der Aushandlung und der Ergreifung repressiver Maßnahmen kann aufgrund der Einbindung der anderen Akteursgruppen mit einer größeren Akzeptanz und Legitimation dieser Schritte gerechnet werden.

Ein Ergebnis kann allerdings auch sein, dass keineswegs zwangsläufig alle bestehenden Regionalisierungsformen und Nutzungssysteme aus dem zukünftigen Naturschutzgebiet ausgeschlossen werden müssen. Die Aushandlung ermöglicht die Anwendung gradueller und kompromissorientierter Ansätze, so dass flexible und jeweils angepasste Lösungen gefunden werden können. In einer sehr optimistischen Interpretation ist dadurch unter Umständen sogar eine Rekonsolidierung von Nutzungssystemen denkbar, die im regionalen Kontext vor der Schutzgebietseinrichtung bereits unter Druck standen oder in der Auflösung begriffen waren. Allerdings hängt der Erfolg solcher Ansätze gerade bei weitgehend machtlosen Akteuren sehr stark vom Aufbau dauerhaft funktionsfähiger und möglichst in übergeordnete Netzwerkstrukturen eingebundener Organisationen ab.

Ein zentrales Element des Szenarios ist sein prozessorientierter Charakter. Letztlich kann kein «Endstadium» erreicht werden, sondern die Interaktion muss als dynamischer Prozess verstanden werden, in dem eine kontinuierliche Aushandlung der unterschiedlichen Naturaneignungen der Beteiligten stattfindet.

Die in Bezug auf die hier betrachteten Fallbeispiele wichtigste Frage, ob grundsätzlich auch aus den zuvor beschriebenen Szenarien ein Übergang in das interaktive Szenario möglich ist, lässt sich letztlich nur von Fall zu Fall, unter Berücksichtigung der spezifischen Besonderheiten, die auch für den Verlauf ihrer jeweiligen Institutionalisierungspfade ausschlaggebend waren, beantworten. Doch auch ohne einen solchen Übergang ist von einer dynamischen Weiterentwicklung der Naturschutzgebiete der Pantanal-Region auszugehen, wobei diese Entwicklung durchaus mit einer Fortsetzung oder Verschärfung der bereits bestehenden Konflikte einhergehen kann.

Der hier gewählte Ansatz der Rekonstruktion von Institutionalisierungspfaden ermöglicht ein umfassenderes
Verständnis der Planung und Einrichtung von Naturschutzgebieten als sozialem und politischen Prozess. Als konfliktträchtig haben sich dabei nicht in erster Linie die hier vorgestellten Grundkomponenten der Institutionalisierung selbst erwiesen, sondern vielmehr ihre jeweilige konkrete Anordnung sowie die Strategien zu ihrer Durchsetzung. Dies eröffnet aber auch die Chance, dass durch einen veränderten Umgang mit den angesprochenen Komponenten im Sinne des vorgestellten Szenarios 4, zwar nicht ein konfliktfreier, aber unter Umständen ein in der Praxis erfolgreicherer Verlauf der Institutionalisierung von Naturschutzgebieten möglich wird.

\section{Literatur}

BLAIKIE, P. (1995): Changing environments or changing views? A political ecology for developing countries. In: Geography 80/3: 203-214.

Blaikie, P. \& S. Jeanrenaud (1997): Biodiversity and human welfare. - In: GHIMIRE, K. \& M. PIMBert (Hrsg.): Social change and conservation. Environmental politics and impacts of national parks and protected areas. - London: Earthscan: 46-70.

BRyant, R. \& S. BaILey (1997): Third world political ecology. - London: Routledge.

Colchester, M. (1997): Salvaging nature: indigenous peoples and protected areas. - In: Ghimire, K. \& M. Pimbert (Hrsg.): Social change and conservation. Environmental politics and impacts of national parks and protected areas. - London: Earthscan: 97-130.

CoY,M.(1991):Sozio-ökonomischer Wandel und Umweltprobleme in der Pantanal-Region Mato Grossos (Brasilien). - In: Geographische Rundschau 43/3: 174-182.

Drumond, M.A. (Hrsg.) (1999): Oficina sobre gestão participativa em unidades de conservação. - Anais: Belo Horizonte.

EllenBERG, L. (1998): Naturschutz und Entwicklungszusammenarbeit, Tourismus/Ressourcenschutz. - In: Rundbrief Geographie 149: 27-29.

Ghimire, K. \& M. Pimbert (1997): Social change and conservation. An overview on concepts and issues. - In: GHImiRe, K. \& M. Pimbert (Hrsg.): Social change and conservation. Environmental politics and impacts of national parks and protected areas. - London: Earthscan: 1-45. Gómez-Pompa, A. \& A. Kaus (1992): Taming the wilderness myth. - In: BioScience 42/4: 271-279.

IUCN (World Conservation Union) (1994): Guidelines for protected areas management categories. - Gland. MMA (Ministério do Meio Ambiente) (2000): SNUC - Sistema Nacional de Unidades de Conservação da Natureza. Lei no 9.985 de 18 de julho de 2000. - Brasília. Müller-Böker, U., Backhaus, N. \& M. Kollmair (1998): Naturkonzepte, Globalisierung des Naturschutzes. - In: Rundbrief Geographie 149: 25-27.

Olwig, K. \& K. Olwig (1979): Underdevelopment and the development of «natural» park ideology. - In: Antipode 11: 16-25. 
PAASI, A. (1986): The institutionalization of regions. A theoretical framework for understanding the emergence of regions and the constitution of regional identities. - In: Fennia 164/1: 105-146.

PeEt, R. \& M. WATTS (1996): Liberation ecologies. Environment, development, social movements. - London: Routledge.

Ramos, A. \& J.P. Capobianco (Hrsg.) (1996): Unidades de Conservação no Brasil. Aspectos legais, experiências inovadores e a nova legislação (SNUC). - = Documentos do ISA 1, São Paulo.

RöPER, M. (1998): Planung und Einrichtung von Naturschutzgebieten aus sozialgeographischer Perspektive. Beispiele aus der Pantanal-Region im Westen Brasiliens. - In: ErdmanN, K.-H., BorK, H.-R. \& K. GrunEWALD (Hrsg.): Geographie und Naturschutz. Beiträge zu einer naturverträglichen Entwicklung. - = MAB-Mitteilungen 45, Bonn: 241-265.

RÖPER, M. (2000): Reconstruindo o histórico do Parque Nacional do Pantanal Matogrossense: Uma contribuição à análise sócio-política das unidades de conservação. - In: Rede Nacional Pró-Unidades de Conservação \& Fundação O Boticário de Proteção à Natureza (Hrsg.): II. Congresso Brasileiro de Unidades de Conservação, Anais, Vol. II: Trabalhos Técnicos, Campo Grande: 452-461.

WERLEN, B. (1997): Sozialgeographie alltäglicher Regionalisierungen. Band 2: Globalisierung, Region und Regionalisierung. - = Erdkundliches Wissen 119, Stuttgart.

\section{Zusammenfassung: Institutionalisierungspfade von Na- turschutzgebieten - Drei Fallbeispiele und ein Modell} In dem Beitrag wird die Entstehung und der Verlauf von Konflikten bei der Planung und Einrichtung von Naturschutzgebieten in Entwicklungsländern durch die Kombination verschiedener sozialgeographischer Ansätze modellhaft erfasst. Naturschutzgebiete werden dabei als eine Form der Institutionalisierung des Zugangs zu und der Kontrolle von Raum begriffen. Die Konflikte sind auf das Aufeinandertreffen zahlreicher Akteure, Interessen, Aneigungs- und Nutzungsmuster sowie Machtkonstellationen zurückzuführen. Der Vergleich von drei Schutzgebieten aus der Pantanal-Region im Westen Brasiliens zeigt, dass der Institutionalisierungsprozess selbst innerhalb eines einheitlichen regionalen Rahmens sehr unterschiedliche Verläufe nehmen kann. Als konfliktträchtig haben sich dabei nicht in erster Linie die Grundkomponenten der Institutionalisierung erwiesen, sondern vielmehr ihre jeweilige konkrete Anordnung sowie die Strategien zu ihrer Durchsetzung. Dies eröffnet aber auch die Chance, dass durch einen veränderten Umgang mit diesen Komponenten zwar nicht ein konfliktfreier, aber unter Umständen ein in der Praxis erfolgreicherer Verlauf der Institutionalisierung von Naturschutzgebieten möglich wird.

\section{Summary: Institutionalization Paths of Protected Areas - Three case studies and a model}

The article presents a social geographical model for the analysis of the conflicts that arise in the planning and implementation of protected areas in developing countries. For this purpose protected areas are perceived as a mode of institutionalization of the access to and the control of space. The conflicts arise both due to the interaction of a variety of actors, interests, modes of spatial appropriation and landuse, as well as to the distribution of power among the actors involved. A comparison of three protected areas located in the Pantanal Region in Western Brazil shows that even within the same regional context the institutionalization processes follow very different paths. The conflicts were much less shaped by the basic components of the institutionalization process than by their respective constellation and their implementation strategies. This implies that the development of new ways of handling the components can lead to the establishment of institutionalization paths, that may not be free of conflict, but possibly more successful in situ.

\section{Résumé: Voies d'institutionalisation des réserves naturelles - trois exemples et un modèle}

L'article propose un modèle socio-géographique pour la compréhension des causes et du déroulement des conflits, qui accompagnent la planification et l'aménagement de réserves naturelles dans les pays en voie de développement. Les réserves naturelles sont perçues comme une forme d'institutionalisation de l'accès à l'espace et de son contrôle. Les conflits qui surgissent sont dus à la présence d'une multiplicité d'acteurs, d'intérêts, de modes d'appropriation et d'utilisation de l'espace, aussi bien qu'aux chances inégales d'accès au pouvoir des différents groupes concernés. La comparaison des trois exemples, pris dans le bassin du Pantanal dans l'ouest brésilien, montre qu'en dépit du même contexte régional le processus d'institutionalisation peut emprunter des voies bien différentes. Les exemples montrent cependant aussi que ce ne sont pas tellement les différents éléments constitutifs de ce processus qui engendrent le conflit, mais plutôt la façon de les aborder et les stratégies choisies pour les imposer aux autres acteurs. Une nouvelle façon d'aborder les différents éléments du processus d'institutionalisation peut donc donner des résultats plus encourageants, en reconnaissant l'existence d'acteurs et de conflits jusqu'à présent sous-estimés.

Monika Röper, Zentralinstitut für Lateinamerika-Studien, Katholische Universität Eichstätt, Ostenstrasse 26, D-85071 Eichstätt.

e-mail:monika_roper@hotmail.com

Manuskripteingang/received/rentrée du manuscrit: 7.2.2001

Annahme zum Druck/accepted for publication/ acceptation à l'impression: 17.4.2001 